05,13

\title{
Влияние амплитуды СВЧ-воздействия на спиновый ток границы платина/железоиттриевый гранат
}

\author{
(C) К.И. Константинян ${ }^{1}$, Г.А. Овсянников ${ }^{1}$, К.Л. Станкевич ${ }^{1}$, Т.А. Шайхулов ${ }^{1}$, В.А. Шмаков ${ }^{1}$, A.A. Климов $^{1,2}$ \\ ${ }^{1}$ Институт радиотехники и электроники им. В.А. Котельникова РАН, \\ Москва, Россия \\ ${ }^{2}$ Российский технологический университет - МИРЭА, \\ Москва, Россия \\ E-mail: gena@hitech.cplire.ru
}

Поступила в Редакцию 9 апреля 2021 г.

В окончательной редакции 9 апреля 2021 г.

Принята к публикации 19 апреля 2021 г.

Исследованы спектры спинового тока в гетероструктуре, состоящей из эпитаксиальной пленки железоиттриевый иттриевого граната $\mathrm{Y}_{3} \mathrm{Fe}_{5} \mathrm{O}_{12}(\mathrm{YIG})$, выращенной на подложке из галлий-гадолиниевого граната $\mathrm{Gd}_{3} \mathrm{Ga}_{5} \mathrm{O}_{12}$ (GGG) и пленки платины $(\mathrm{Pt})$. Измерения спинового тока, вызванного СВЧ-воздействием на YIG пленку в режиме ферромагнитного резонанса и обратным спиновым эффектом Холла, проводились при вариации мощности СВЧ-воздействия $20 \mu \mathrm{W}-50 \mathrm{~mW}$ и частоты $2-9 \mathrm{GHz}$ в температурном диапазоне $T=77-300 \mathrm{~K}$ с целью выявления роли спин-волновых резонансов в YIG на спектральные характеристики спинового тока. Обнаружено, что при увеличении мощности СВЧ-воздействия на частотах $f=2-3 \mathrm{GHz}$ aмплитуда спинового тока, вызванная спин-волновыми резонансами поверхностных спиновых волн, становится сравнимой с вкладом от ферромагнитного резонанса.

Ключевые слова: ферромагнитный резонанс, спин-волновой резонанс, спиновый ток, железоиттриевый гранат, платина.

DOI: $10.21883 /$ FTT.2021.09.51258.23H

\section{1. Введение}

Известно, что в гетероструктурах, состоящих из ферромагнетика (FM) и нормального (немагнитного) металла (NM) в условиях ферромагнитного резонанса (FMR) поперек их границы возникает спиновый ток $I_{S}$, который определяет величину зарядового тока в нормальном металле $I_{Q}$ вследствие обратного спинового эффекта Холла:

$$
I_{Q}=(e / h) \theta_{S H}\left[\mathbf{n} \times I_{S}\right],
$$

где $\theta_{S H}-$ спиновый угол Холла, $\mathbf{n}-$ единичный вектор спинового момента в ферромагнетике. Экспериментально измеряется напряжение $V_{Q}=R I_{Q}$ $(R-$ сопротивление пленки $\mathrm{Pt})$, возникающее в $\mathrm{NM}$ металле за счет обратного спинового эффекта Холла (ISHE) [1-3]. В гетероструктурах Pt/YIG при СВЧвоздействии спектр $V_{Q}(H)$ наряду с особенностью, вызванной FMR (волновой вектор $k=0$ ) может содержать особенности, вызванные спин-волновыми резонансами (SWR) с $k \neq 0$ [4-6]. Обычно SWR возникают при достаточно большой толщине пленки YIG и невысоких частотах СВЧ-воздействия [4-6]. В данной работе, изменяя мощность СВЧ-воздействия $P_{i n}$, было экспериментально изучено влияние SWR, вызванных поверхностными (MSSW) и объемными (BVSW) спиновыми волнами, на спектр напряжения $V_{Q}(H)$, вызванного протеканием спинового тока. Исследовалось также поведение амплитуды и ширины линии спектра $V_{Q}(H)$ с уменьшением температуры от комнатной до азотной $T=77 \mathrm{~K}$.

\section{2. Образцы и техника измерений}

Пленки железоиттриевого граната $\mathrm{Y}_{3} \mathrm{Fe}_{5} \mathrm{O}_{12}$ (YIG) толщиной $d_{\mathrm{YIG}}=8 \mu \mathrm{m}$ выращивалась с помощью молекулярной эпитаксии на обе стороны подложки (111) $\mathrm{Gd}_{3} \mathrm{Ga}_{5} \mathrm{O}_{12}$ (GGG) толщиной $0.5 \mathrm{~mm}$ и размером в плане $4 \times 3 \mathrm{~mm}^{2}$. Пленка $\mathrm{Pt}$ толщиной $d_{\mathrm{Pt}}=10 \mathrm{~nm}$ напылялась на одну сторону структуры YIG/GGG с помощью распыления на постоянном токе при комнатной температуре.

Магнитная петля гистерезиса YIG-пленки, измеренная с помощью меридионального эффекта Керра [7], имела коэрцитивную силу $H_{C}=30$ Ое и поле насыщения $H_{S}=230 \mathrm{Oe}$. При вращении гетероструктуры относительно нормали к плоскости подложки не было обнаружено магнитной анизотропии.

Исследуемые образцы располагались в держателе так, чтобы СВЧ-магнитное поле $h_{r f}$ было направлено параллельно направлению возникновения напряжения $V_{Q}$ на пленке Pt. Внешнее постоянное магнитное поле $H$ находилось в плоскости подложки и было направлено перпендикулярно $h_{r f}$. Регистрация напряжения $V_{Q}$ проводилась малошумящим синхронным усилителем при модуляции СВЧ-воздействия. Спектр FMR определялся либо из зависимости от магнитного поля отраженного СВЧ-сигнала в широком интервале СВЧ $f=2-18 \mathrm{GHz}$, либо по зависимостям производной $d P / d H$ от $H$ на частотах $f=2-5 \mathrm{GHz}$. 


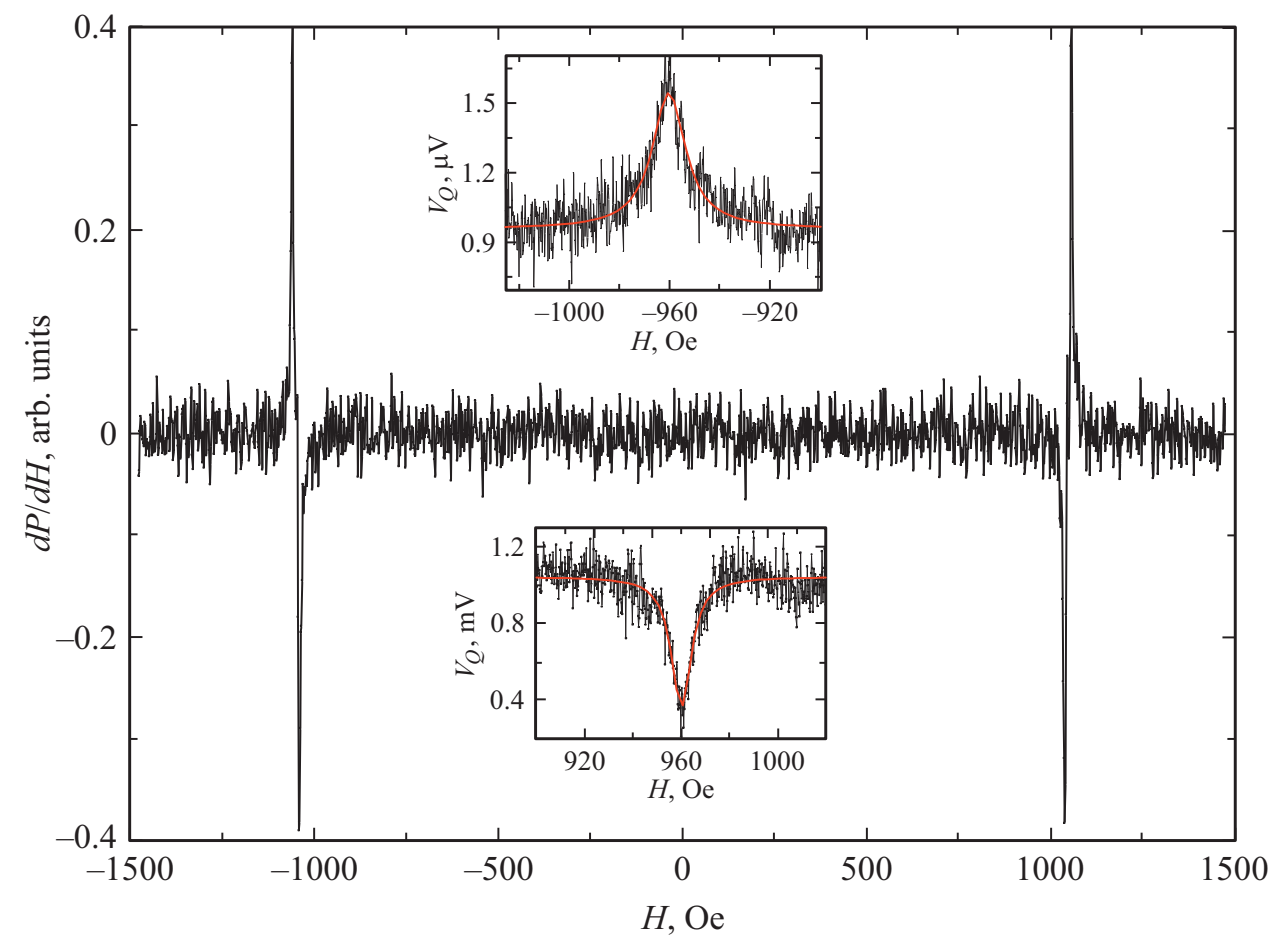

Рис. 1. Зависимость спектра FMR $d P / d H$ от магнитного поля $H, T=78 \mathrm{~K}, f=5 \mathrm{GHz}$. На вставках - спектры $V_{Q}(H)$, снятые при сдвиге частоты СВЧ-воздействия накачки $f=4.74 \mathrm{GHz}$ для двух направлений магнитного поля. Сплошными линиями на вставках показана аппроксимация $V_{Q}(H)$ линиями Лоренца для $k=0$. Мощность СВЧ-воздействия не превышала $3 \mathrm{~mW}$.

\section{3. Экспериментальные результаты}

\section{1. Спиновая проводимость границы Pt/YIG}

При СВЧ-воздействии в режиме FMR в пленке YIG через границу $\mathrm{Pt} / \mathrm{YIG}$ протекает спиновый ток, который определяется спиновой проводимостью границы $\mathrm{Pt} / \mathrm{YIG}$ $g^{\uparrow \downarrow}$ и амплитудой СВЧ-магнитного поля $h_{r f}[3,8,9]$ :

$$
I_{S}=(h f / 4 \pi) g^{\uparrow \downarrow}\left(h_{r f} / 2 \Delta H_{\mathrm{Pt} / \mathrm{YIG}}\right)^{2},
$$

где $\Delta H_{\mathrm{Pt} / \mathrm{YIG}}$ - ширина линии FMR гетероструктуры $\mathrm{Pt} / \mathrm{YIG}$. При однородном уширении линии поглощения $g^{\uparrow \downarrow}$ экспериментально определяется утечкой спинов через границу $[3,5,8,10]$ :

$$
g^{\uparrow \downarrow}=\frac{\gamma M t_{\mathrm{YIG}}}{\mu_{\mathrm{B}} f}\left(\Delta H_{\mathrm{Pt} / \mathrm{YIG}}-\Delta_{\mathrm{YIG}}\right),
$$

где $\gamma$ - гиромагнитное отношение, $M-$ намагниченность пленки $\mathrm{YIG}, t_{\mathrm{YIG}}$ - толщина пленки $\mathrm{YIG}$, $\mu_{\mathrm{B}}$ - магнетон Бора, $\Delta H_{\mathrm{YIG}}$ и $\mathrm{YIG}, \Delta H_{\mathrm{Pt} / \mathrm{YUG}}$ ширины линии FMR пленки YIG и гетероструктуры Pt/YIG соотвественно. Используя (3) для частоты $f=7 \mathrm{GHz}$, получаем $g^{\uparrow \downarrow}=1.1 \cdot 10^{20} \mathrm{~m}^{-2}$. Для сравнения в [5] для границы Pt/YIG получено существенно большее значение $g^{\uparrow \downarrow}=4.8 \cdot 10^{20} \mathrm{~m}^{-2}$, что, возможно, вызвано присутствием других механизмов уширения линии кроме однородного уширения, которое учитывается в соотношении (3). Однако наши оценки вкладов в $g \uparrow \downarrow$ неоднородности ферромагнетика, одномерной спиновой проводимости пленки $\mathrm{Pt}$, двухмагнонного рассеяния и вихре- вого тока показали, что вклады перечисленных факторов в спиновую проводимость не превышают 10\% [11,12].

\section{2. Спектр спинового тока}

На рис. 1 показана зависимость спектра $d P / d H(H)$ гетероструктуры $\mathrm{Pt} / \mathrm{YIG}$ при $T=78 \mathrm{~K}$ и $f=5 \mathrm{GHz}$. Видно, что $d P / d H(H)$ гетероструктуры Pt/YIG имеет симметричный характер с одинаковым знаком поглощения

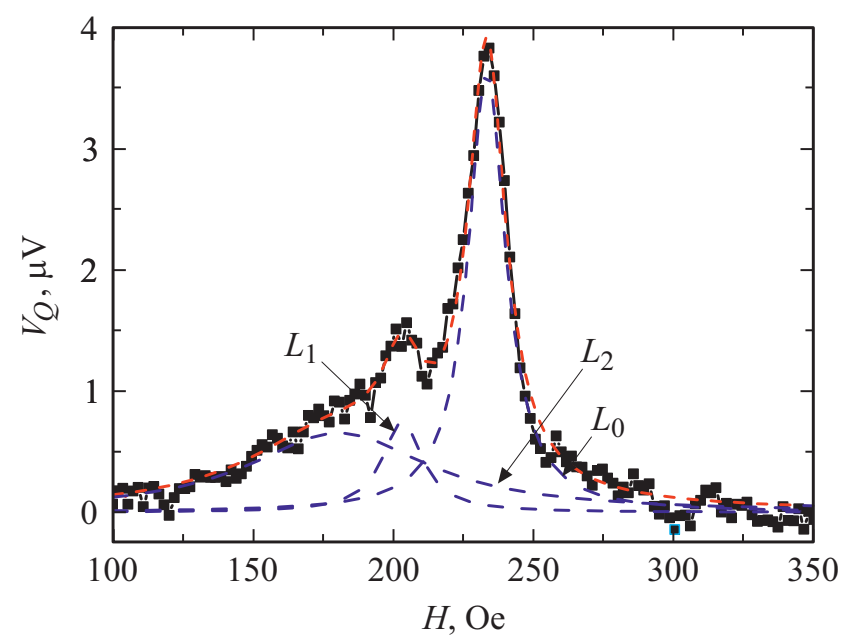

Рис. 2. Зависимость $V_{Q}(H), \quad T=120 \mathrm{~K}, \quad f=2.1 \mathrm{GHz}$, $P_{i n}=2 \mathrm{~mW}$. Эксперимент - квадраты, сплошная красная линия - сумма трех линий Лоренца, (штриховая лииния). 

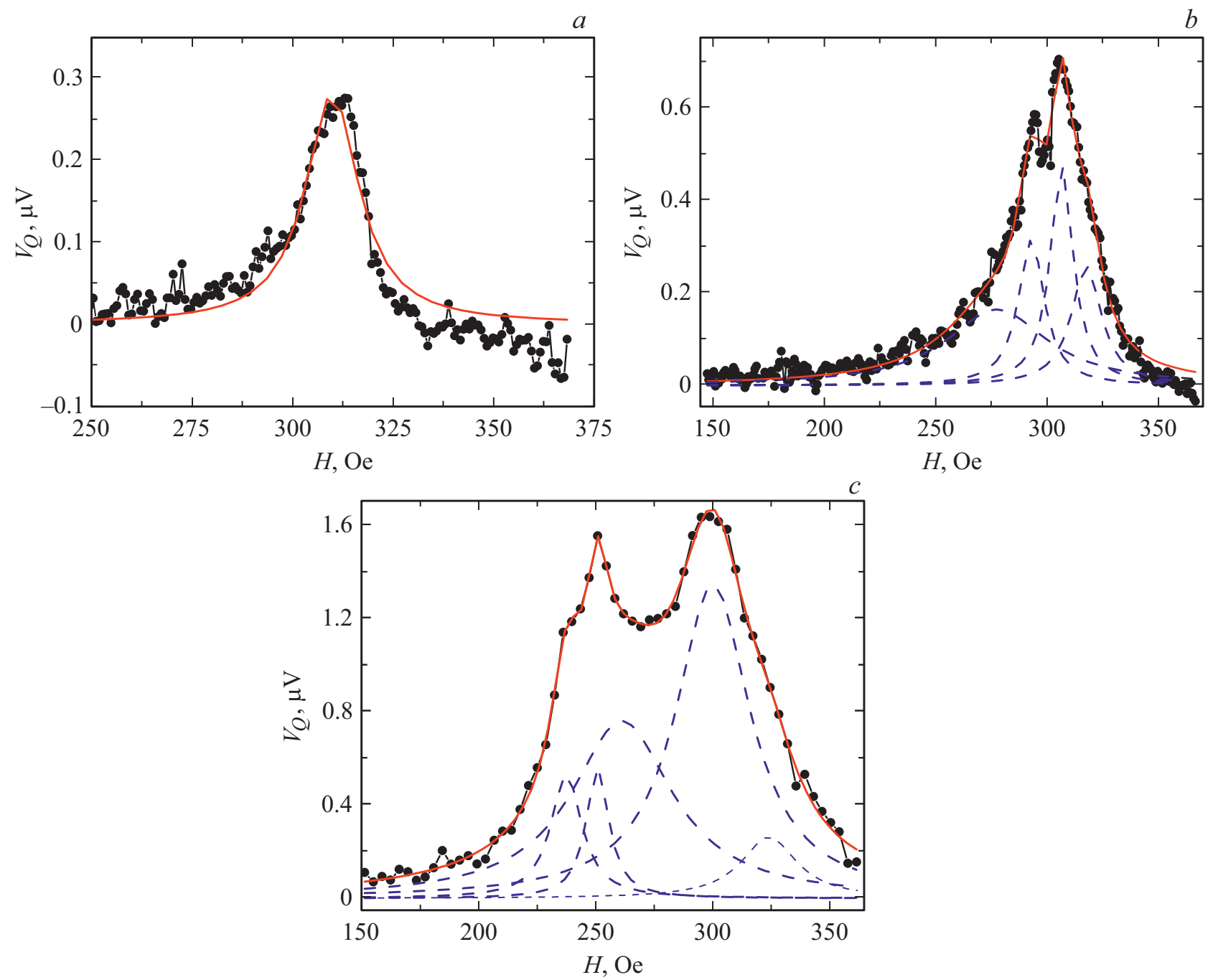

Рис. 3. Зависимости $V_{Q}(H)$ при $T=300 \mathrm{~K}, f=2.1 \mathrm{GHz}$ при выходной мощности СВЧ-воздействия $(a) P_{\text {in }}=2 \mathrm{~mW}-$ аппроксимация одной линией Лоренца, $(b) P_{i n}=10 \mathrm{~mW}-$ аппроксимация четырьмя линиями Лоренца, (c) $50 \mathrm{~mW}-$ аппроксимация пятью линиями Лоренца.

при изменении направления (знака) магнитного поля. На вставках к рис. 1 приведены зависимости $V_{Q}(H)$ для двух значений направления магнитного поля, снятые при небольшой отстройке частоты $f=4.74 \mathrm{GHz}$. В отличие от FMR спектры $V_{Q}(H)$ асимметричны относительно изменения знака $H$ в соответствии с соотношениями $(1,2)$. Сдвиг по полю пиков $V_{Q}$ при $f=4.74 \mathrm{GHz}$ относительно FMR-спектра при $f=5 \mathrm{GHz}$ хорошо соответствует соотношению Киттеля для изотропного случая $f=\gamma\left[H_{0}\left(H_{0}+4 \pi M\right)\right]^{1 / 2}$ при намагниченности $4 \pi M=2.0 \pm 0.01 \mathrm{kG}$ для $T=78 \mathrm{~K}$.

На вставках к рис. 1 приведены спектральные линии $V_{Q}(H)$, аппроксимированные линией Лоренца с $i=0$ :

$$
L_{i}(H)=A_{i} /\left[1+\left[\left(H-H_{i}\right) / \Delta H_{i}\right]^{2},\right.
$$

где $A_{i}-$ амплитуда, $\Delta H_{i}-$ полуширина линии на полувысоте, $H_{i}$ - сдвиг пика $i$-той линии относительно основной $i=0$, соответствующей FMR.
C понижением частоты и увеличением мощности СВЧ-воздействия форма спектра $V_{Q}(H)$ претерпевает искажение и отличается от линии Лоренца. Пример зависимости $V_{Q}(H)$, снятой при температуре $T=120 \mathrm{~K}$ приведен на рис. 2. Видно, что зависимость $V_{Q}(H)$ асимметрична относительно максимума. Достаточно хорошее соответствие формы спектра получается при аппроксимации суммой трех линий Лоренца (4) $L_{i}$ с $i=0,1,2$ (штриховые линии на рис. 2). Заметим, появление в спектре $V_{Q}(H)$ боковых линий обусловлено возникновением SWR: при $H_{i}<H_{0}$ поверхностными (MSSW), а при $H_{i}>H_{0}$ объемными (BVSW) спиновыми волнами [4-6]. Из приведенного рисунка видно, что в данном случае искажение формы спектральной линии $V_{Q}(H)$ происходит при $H<H_{0}$ и, следовательно, обусловлено SWR поверхностных волн. Отметим, что на более высоких частотах искажение спектра $V_{Q}(H)$ не наблюдается, а на FMR спектре отсутствуют SWRособенности. 


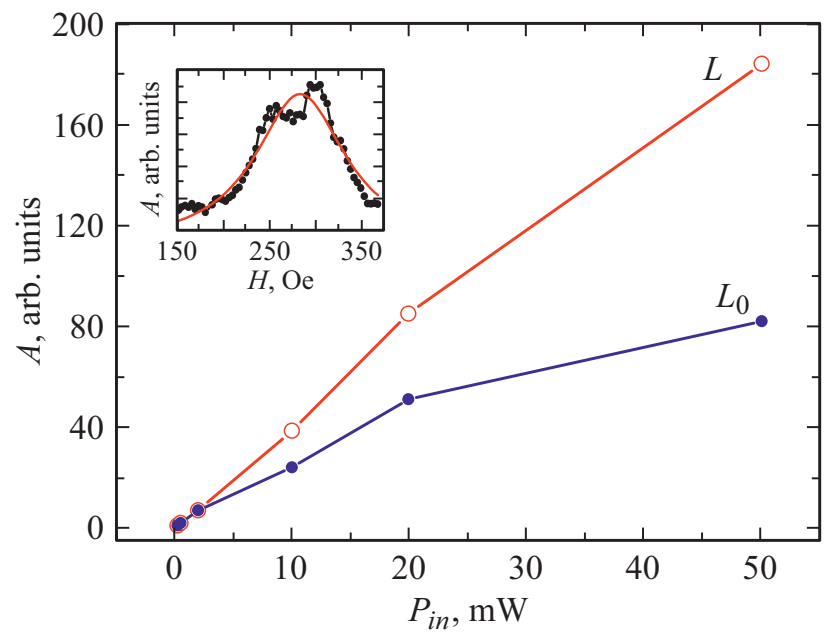

Рис. 4. Зависимости от мощности СВЧ-воздействия амплитуды линии Лоренца FMR компоненты $L_{0}$ (см. рис. 2) и амплитуды, полученной аппроксимацией $V_{Q}(H)$ одной линией (см. вставку к рисунку).

\section{3. Влияние мощности СВЧ-воздействия}

Для определения влияния мощности СВЧ-воздействия на спектр спинового тока были сняты зависимости $V_{Q}(H)$, варьируя подаваемую мощность СВЧ-воздействия $P_{i n}$. На рис. 3 представлены зависимости $V_{Q}(H)$ при $T=300 \mathrm{~K}$ для трех значений $P_{i n}$. Экспериментальную зависимость $V_{Q}(H)$ удается описать совокупностью от одной до пяти линий Лоренца в зависимости от величины $P_{i n}$. При малом значении $P_{i n}=2 \mathrm{~mW}$ наблюдается небольшое отличие спектра $V_{Q}(H)$ от линии Лоренца с $i=0$ (рис. 2). При высокой мощности СВЧ-воздействия присутствуют компоненты $V_{Q}(H)$, вызванные SWR как поверхностными, так и объемными волнами (рис. $3, c$ ). Так, для $P_{\text {in }}=50 \mathrm{~mW}$ помимо $L_{0}$ с $H_{0}=300$ Ое и $\Delta H=38$ Ое, есть три компоненты от поверхностных волн: $L_{1}$ с $H_{1}=261$ Ое и $\Delta H=52 \mathrm{Oe}, L_{2}$ с $H_{2}=251$ Ое и $\Delta H_{2}=11 \mathrm{Oe}, L_{3}$ с $H_{3}=238 \mathrm{Oe}$ и $\Delta H_{2}=16 \mathrm{Oe}$, а также $L_{1}^{*}$ от объемной волны с $H_{1}^{*}=324 \mathrm{Oe}$ и $\Delta H_{1}^{*}=29 \mathrm{Oe}$. Отметим, что аппроксимация одними лишь линиями Лоренца дает хорошее совпадение с экспериментом, но не учитывает вклад дисперсионной (асимметричной) составляющей FMR [13].

На рис. 4. приведены две зависимости от $P_{\text {in }}$ параметра $A$, пропорционального произведению амплитуды спектральной линии $V_{Q}(H)$ на ширину линии. Зависимость, обозначенная $L$ соответствует аппроксимации всей кривой $V_{Q}(H)$ одной линией Лоренца (см. вставку на рис. 4), а зависимость $L_{0}$ соответствует значению $A$ линии Лоренца с $i=0$ при разложении спектра $V_{Q}(H)$ на составляющие (см. рис. 3 ). Видно, что зависимости $L$ и $L_{0}$ расходятся при $P_{i n} \approx 10 \mathrm{~mW}$. Такое поведение параметра $A$ свидетельствует о том, что с увеличением $P_{\text {in }}$ наблюдается перераспределение доминирующих механизмов генерации спинового тока от FMR к SWR.

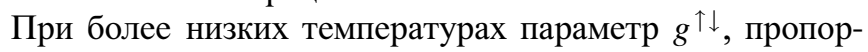
циональный $M$ растет и вклад в спектр $V_{Q}(H)$ SWR поверхностных волн проявляется отчетливее, как видно из сравнения данных на рис. 2 и 3, а, полученных при одинаковой мощности СВЧ-воздействия $P_{i n}=2 \mathrm{~mW}$.

\section{4. Температурная зависимость спинового тока}

При изменении температуры амплитуда напряжения $V_{Q}(T)$ изменяется из-за температурных зависимостей намагниченности $M(T)$, которая определяет $g^{\uparrow \downarrow}$, ширины линии спектра спинового тока, спинового угла $\theta_{S H}$, а также от мощности СВЧ-воздействия, $h_{r f}^{2}$ (см. соотношения (1)-(3)).

На рис. 5 приведена температурная зависимость ширины линии $\Delta H$ и амплитуды тока $I_{Q}=V_{Q} / R$, пересчитанная с учетом сопротивления пленки $\mathrm{Pt} R(T)$. Видно, что для случая $f=3 \mathrm{GHz}$ и $P_{\text {in }}=2 \mathrm{~mW}$ ширина линии $\Delta H$ в пределах экспериментальной погрешности не зависит от температуры. Хотя температура Кюри YIG существенно выше комнатной, зависимость $M(T)$, полученная из экспериментальных данных $H_{0}(T)$ по формуле Киттеля для изотропного ферромагнетика, нарастает с уменьшением температуры и выходит на плато при $T \leq 100$ К. Такое поведение типично для YIG-пленок, в том числе и с внесенными неоднородностями [14]. В наших гетероструктурах величина намагниченности $M$ при комнатной температуре составляет $2 / 3$ от $M$ при $T=120 \mathrm{~K}$. Соответственно, без учета остальных факторов, влияющих на температурную зависимость $I_{Q}(T)$, должен наблюдаться спад $I_{Q}$ с увеличением температуры. Однако в эксперименте на $f=2.1 \mathrm{GHz}$, напротив, наблюдался рост $I_{Q}$ с температурой. Возможным объяснением может служить зависимость от температуры импедансных характеристик интерфейса YIG/Pt, влияющих на величину $h_{r f}$ (см. соотношение в (2)). Более подробные температурные измерения $I_{Q}(T)$ были проведены на частоте $f=3 \mathrm{GHz}$, представленные на рис. 5. Видно, что амплитуда $I_{Q}(T)$, аппроксимированная

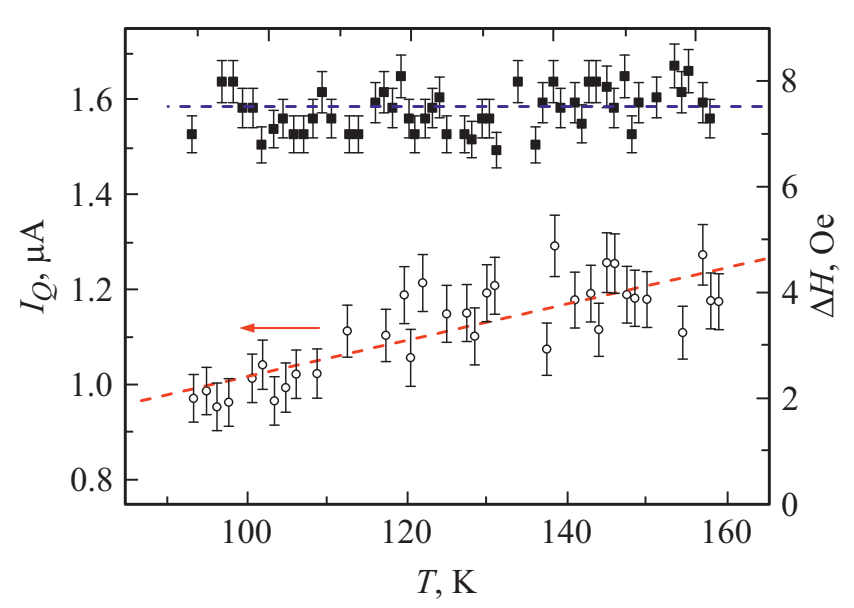

Рис. 5. Температурные зависимости ширины линии $\Delta H$ (квадраты) и амплитуды тока $I_{Q}$ (круги), $P_{i n}=2 \mathrm{~mW}, f=3 \mathrm{GHz}$. Линейные аппроксимации зависимостей показаны штрихами. 
в приближении наименьших квадратов линейной зависимостью, демонстрирует рост с увеличением температуры. Заметим, рост амплитуды напряжения $V_{Q}(T)$ с температурой был получен также в работе [6] на частоте $f=9.12 \mathrm{GHz}$.

\section{4. Заключение}

Наличие спин-волновых резонансов (SWR) искажает спектр спинового тока Pt/YIG гетероструктур. На относительно низких частотах $(3 \mathrm{GHz}$ и ниже) при малых мощностях накачки $P_{i n}<2 \mathrm{~mW}$ вклад в спектральную линию $V_{Q}(H)$ от SWR существенно меньше, чем от FMR. C увеличением мощности СВЧ-воздействия $P_{\text {in }}$ влияние SWR становится заметнее, особенно тех резонансов, которые обусловлены поверхностными волнами. Зависимость спектральных характеристик спинового тока от $P_{\text {in }}$ свидетельствует о перераспределении мощности накачки от FMR к SWR. C уменьшением температуры амплитуда тока $I_{Q}$, обусловленного ISHE уменьшается, хотя величина намагниченности $M$ растет с понижением температуры. При этом изменения в ширине линии $I_{Q}(H)$ не обнаруживаются.

\section{Благодарности}

Авторы благодарны В.А. Ацаркину, В.В. Демидову, Ю.В. Кислинскому, А.М. Петржику и А.В. Шадрину за полезное обсуждение полученных результатов и помощь при проведении измерений.

\section{Финансирование работы}

Работа выполнена в рамках государственного задания и частично поддержана Российским фондом фундаментальных исследований (проектами РФФИ 18-57-16001, 19-07-00143). Работа А.А. Климова частично поддержана Российским научным фондом, проект 20-12-00276.

\section{Конфликт интересов}

Авторы заявляют, что у них нет конфликта интересов.

\section{Список литературы}

[1] M.I. Dyakonov, V.I. Perel. Phys. Lett. A 35, 459 (1971).

[2] E. Saitoh, M. Ueda, H. Miyajima, G. Tatara. Appl. Phys. Lett. 88, 182509 (2006)

[3] Y. Tserkovnyak, A. Brataas, G.E.W. Bauer. Phys. Rev. Lett. 88, 117601 (2002).

[4] C.W. Sandweg, Y. Kajiwara, K. Ando, E. Saitoh, B. Hillebrands. Appl. Phys. Lett., 97, 252504 (2010).

[5] S.M. Rezende, R.L. Rodríguez-Suárez, M.M. Soares, L.H. Vilela-Leão, D. Ley Domínguez, A. Azevedo. Appl. Phys. Lett. 102, 012402 (2013).

[6] Se. Dushenko, Yukio Higuchi, Yuichiro Ando, Teruya Shinjo, Masashi Shiraishi. Appl. Phys. Exp. 8, 103002 (2015).
[7] A.S. Grishin, G.A. Ovsyannikov, A.A. Klimov, V.V. Demidov, K.Y. Constantinian, I.V. Borisenko, V.L. Preobrazhensky, N. Tiercelin, P. Pernod. J. Electron. Mater. 47, 1595 (2018).

[8] Fengyuan Yang, P. Chris Hammel. J. Phys. D 51, 253001 (2018).

[9] O. Mosendz, V. Vlaminck, J.E. Pearson, F.Y. Fradin, G.E.W. Bauer, S.D. Bader, A. Hoffmann. Phys. Rev. B 82, 214403 (2010).

[10] S. Emori, U.S. Alaan, M.T. Gray, V. Sluka, Y. Chen, A.D. Kent, Y. Suzuki. Phys. Rev. B 94, 224423 (2016).

[11] Т.А. Шайхулов, Г.А. Овсянников. ФТТ 60, 11, 2160 (2018).

[12] T.G.A. Verhagen, H.N. Tinkey, H.C. Overweg, M. van Son, M. Huber, J.M. van Ruitenbeek, J. Aarts. J. Phys. Condens. Matter 28, 056004 (2016).

[13] M. Harder, Z.X. Cao, Y.S. Gui, X.L. Fan, C.-M. Hu. Phys. Rev. B 84, 054423 (2011).

[14] I.H. Solt Jr. Appl. Phys. A 33, 1189 (1982).

Редактор Д.В. Жуманов 